\title{
Guest Editorial: Selected contributions from the 16th European Conference on the Mathematics of Oil Recovery (ECMOR XVI)
}

\author{
Dayal Gunasekera ${ }^{1} \cdot$ James Bennett ${ }^{1}$
}

Published online: 13 March 2020

(C) Springer Nature Switzerland AG 2020

The 16th European Conference on the Mathematics of Oil Recovery, better known as ECMOR XVI, was held during 3-6 September 2018 at the World Trade Center Barcelona, on the sea front and a few minutes of walk from the historic city. The conference was a resounding success, with a high quality of technical content, enjoyable networking events, high-calibre facility and the highest ever participation of 228 delegates. A total of 88 oral presentations and 88 poster presentations were given on a wide variety of topics in mathematical modelling, science and technologies applied to hydrocarbon exploration, development and production.

Since the inaugural conference in Cambridge UK, three decades ago, ECMOR has been convened every 2 years, gathering applied mathematicians and geoscience engineers from both academia and industry to discuss recent advances in the mathematics of oil recovery. The conference attracts over 200 delegates, providing both informal and more structured opportunities for discussion and exchange of ideas between world specialists in simulation, modelling and data integration.

Technical presentations at ECMOR XVI consisted of two parallel tracks, one focusing on physical models with sessions titled: Reservoir Characterization, CO2 Sequestration,
Enhanced Oil Recovery, Reservoir Geomechanics, Reservoir Geochemistry, Fracture Simulation, Unconventionals and Risk and Uncertainty. The other track focused on numerical topics with the following sessions: Discretization, Linear Solvers, Non-linear Solvers, Well and Facility Optimization, Data Assimilation and Machine Learning and Proxy Models. Full proceedings of the conference are available electronically via the EAGE conference paper repository EarthDoc: http:// earthdoc.eage.org.

As with previous ECMOR conferences, a selection of refereed and revised papers is published in a Special Issue of the Computational Geosciences journal dedicated to ECMOR XVI. We would like to thank all authors and reviewers of the submitted papers and members of ECMOR XVI Program Committee, whose valuable time and expertise ensured the high quality of this Special Issue. We believe that this collection of 36 papers provides an excellent illustration of the breadth and depth of ECMOR XVI, and as such the state of the art of mathematics of oil recovery, and we hope that it proves a useful resource to the readers of Computational Geosciences.
Dayal Gunasekera

dlg@slb.com

James Bennett

jim@slb.com

1 Schlumberger Ltd., Abingdon, Oxfordshire, UK 